\title{
Research on E-Commerce Platform of Airport Economic Zone
}

\author{
Yang Changhui*
}

School of Business, Zhengzou University, Zhengzhou, Henan, 450001, P.R. China

\begin{abstract}
Information technology has been widely used in the enterprise management of airport economic zone. The use of information technology in constructing apparel industry e-commerce platform can realize the electronic and informatization in the trading and management and that can realize intelligent decision support, standardization of business process and automation of interactive information release. In the article, the necessity of apparel industry e-commerce platform construction is analyzed, and then discusses the overall structure and network topology structure of the e-commerce platform, analyses the design and construction of information platform, and finally discusses the main application system of information platform. Platform application helps to strengthen the institutionalization and standardization of enterprise management, standardize the market economic order, and improve product quality and service quality. Moreover, it helps to improve the competitiveness of the industry, enterprises and products in the airport economic zone.
\end{abstract}

Keywords: Airport economic zone, apparel industry, e-commerce platform, network structure, system structure.

\section{INTRODUCTION}

At present, under the trend of economic globalization and regional economic integration, the world science and technology changes with each passing day, the competition in overall national strength is becoming increasingly fierce. Using the modern information technology to improve trading efficiency, reduces transaction costs, improves enterprise competitiveness and has become an effective way to implement trade facilitation. Airport economic zone apparel industry e-commerce platform involves trade, finance, customs, taxation, insurance, logistics and so on. E-commerce platform construction of clothing industry helps to realize electronic trading, improves transaction efficiency, reduces the transaction cost, promotes the interaction of information flow, cash flow and logistics in the airport economic zone, and helps to promote the competitiveness of the enterprise.

Construction of apparel industry e-commerce platform in the airport economic zone relies on existing sales and management system. We should increase the intangible market construction as the electronic commerce, dredge the online trading channel, provide information services and management services for the majority of merchants, gradually guide the general merchants to build e-commerce transaction mode, realize the organic unity between tangible market and e-commerce, further enhance the level of management and speed up the promotion from commerce form to the business form. With the combination of brand, information and logistics, and the combination of exhibition and orders, enterprise competitiveness will gradually improve $[1,2]$.

Apparel industry e-commerce platform construction is a systematic engineering. Speaking from the organization form, in the economic zone who engaged in the apparel business belong to small and medium-sized enterprises; they cannot have an independent information system construction. In order to promote e-commerce applications, it is necessary to construct the public Internet trading platform, solve business network access, data hosting and security problems. To carry out the electronic commerce, one cannot leave a safe and efficient means of electronic payment [3]. That needs to build online banking for local users and online payment platform for the Internet users. In business services, the main construction contents of the airport economic zone apparel industry e-commerce platform should include:

(1) The Internet web site: for information release and Internet business bearing

(2) The member service system: to provide enterprise information, product information release and trade matching services for clothing enterprises

(3) The network virtual shop: to provide online virtual store service for the small retailers

(4) Raw material bidding procurement platform: to serve for the garment production enterprises and raw material suppliers

(5) Mobile commerce systems: a small business management system that uses mobile communication technology and provides service for brands and brand name dealerships

(6) Logistics information system: to provide public logistics information service

In order to promote the exchange of information and services, we also need to build a network business service platform. To improve management and service level, improve the management order, establish credit system, we should popularize the market management system application. 


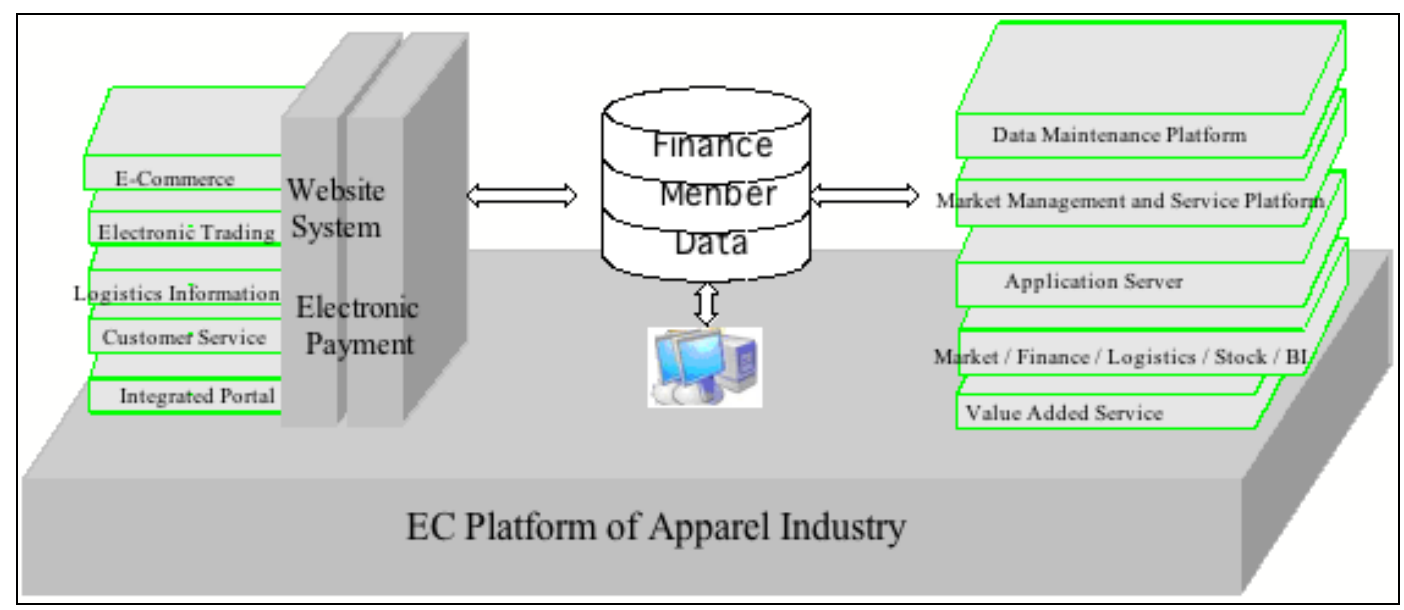

Fig. (1). Ssystem structure diagram of apprel industry e-commerce platform.

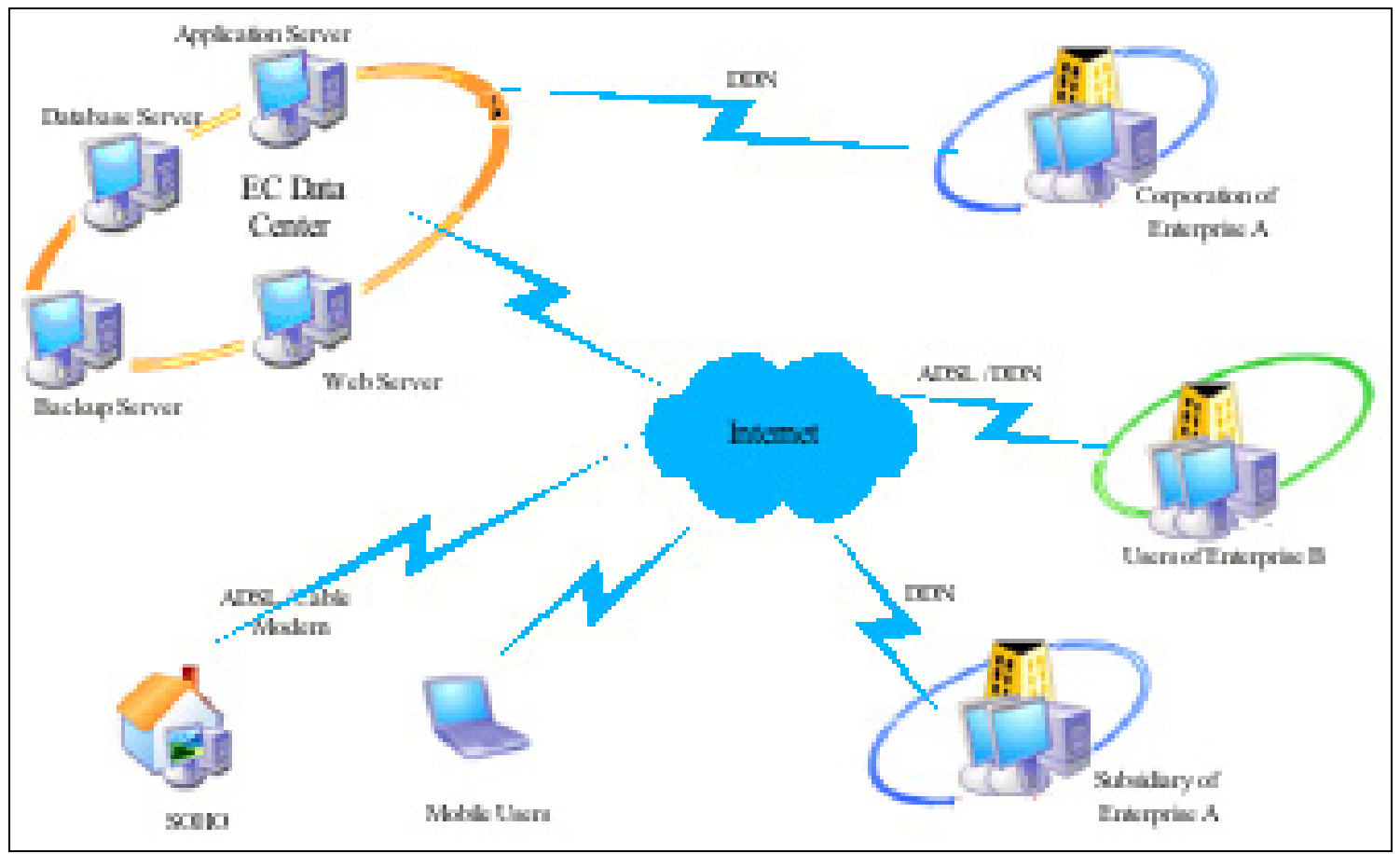

Fig. (2). System network structure diagram of apparel industry e-commerce platform.

\section{SYSTEM FRAMEWORK OF E-COMMERCE PLATFOR}

E-commerce platform system structure is shown in Fig. (1), the network structure as shown in Fig. (2), details as follows.

All modules of apparel industry e-commerce Platform construction are based on J2EE. Java 2 Platform, Enterprise Edition (J2EE) is dominated by SUN, and start with IBM, BEA Systems, Oracle and other manufacturers, and is widely recognized by industry standards, and it consists of the Servlet/JSP, applets, Java Bean and JDBC, JMS and JNDI, JTA, JAXP and a series of technology. J2EE provides a component-based approach to design, develop, assemble and lay out enterprise applications [3]. J2EE platform provides a distributed application model that has a multilevel structure, this model has the ability to reuse components, data exchange based on extensible markup language (XML), with the unified security mode and flexible transaction control. J2EE platform has many characteristics, such as good portability, powerful functions, high efficiency performance, safety, high expansibility and flexibility [4]. It is the current mainstream WEB, enterprise application development platform.

E-commerce system adopts hierarchical structure and modularization management to reduce the complexity of the system and improve the system scalability.

It uses the Hibernate technology in the hierarchical structure, and increase the persistence layer on the basis of the original three layered structure, to realize the unified management to the persistence operations [5].

\section{NETWORK INFRASTRUCTURE OF E-COMME- RCE PLATFORM}

Network infrastructure of the apparel industry ecommerce platform of the airport economic zone includes 


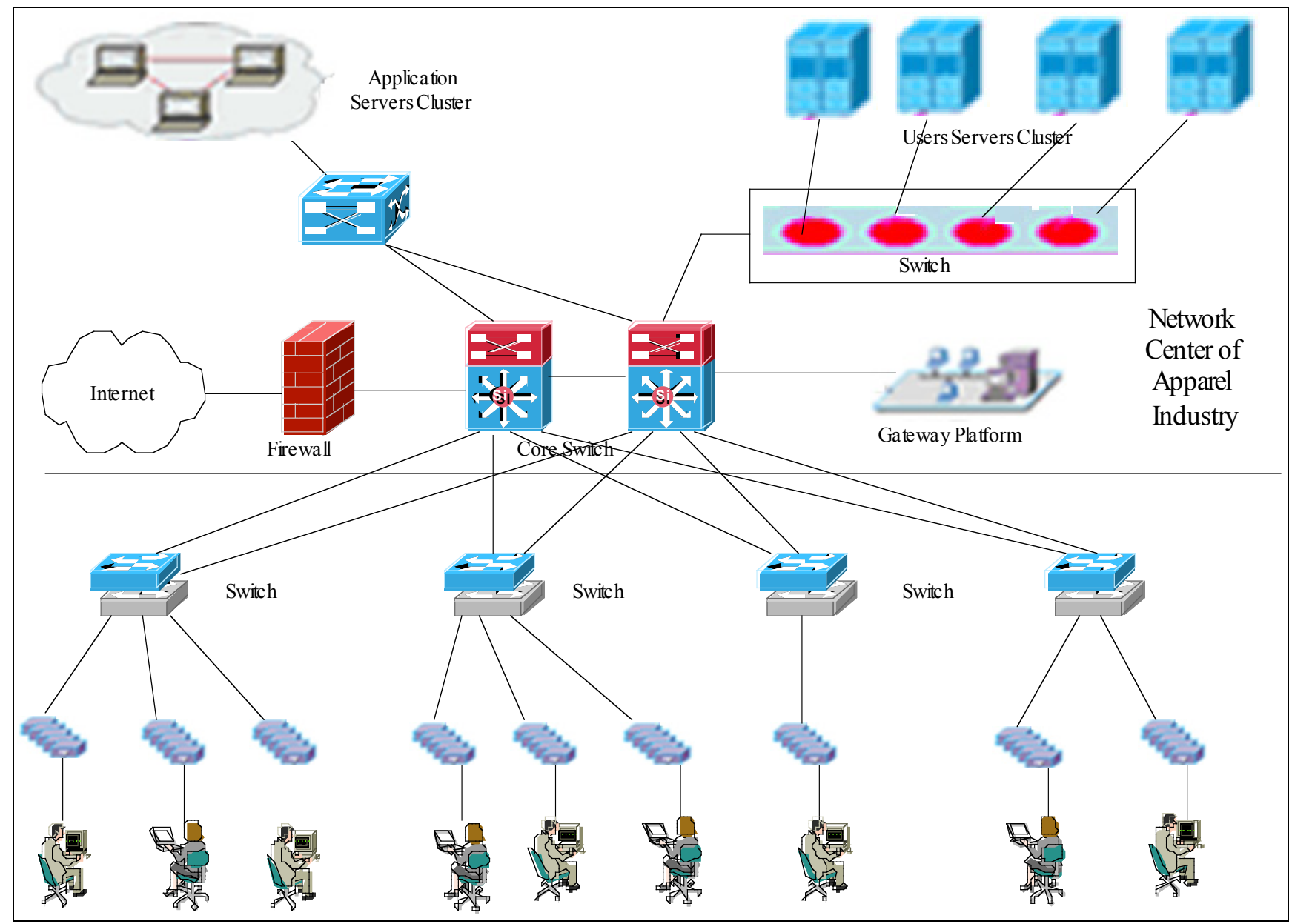

Fig. (3). Metropolitan area network topology figure.

three aspects: metropolitan area network, Internet data center and network security facilities.

\subsection{Main Construction}

Metropolitan area network is a three layered that network that covers the entire area and look the data center as the tandem center. It mainly includes the backbone network and market intranet two parts, metropolitan area network topology is shown in Fig. (3).

\subsection{Backbone Network}

The construction of data backbone network adopts the mature and advanced metropolitan area network technology. It supports TCP/IP protocol, supports QoS, MPLS, security, VPN, VLAN, multicast, support network computer's highly depending on the network, and supports a variety of multimedia data services that are based on TCP/IP (e.g., IP telephone, Intranet) $[6,7]$. Network topology structure is shown in Fig. (4).

According to the demand of the business system, application system and management departments, business and customers in the information construction, the backbone network construction must ensure the cable to be connected to the market and must ensure that the network bandwidth is not less than $1 \mathrm{Gbps}$, and it has the ability to be extended to dozens of Gbps.

\subsection{Market Intranet}

Market internal network mainly adopts 100 MBPS Ethernet to exchange to the desktop (merchants), the network level is between convergence layer and access layer, the specific level can be determined according to different markets and the physical situation. Market internal network can operate closely; at the same time can also be divided into different VLAN.

For some wiring difficult market, in the access layer, we can consider using ADSL access (the demand of the network computer application to the network is higher, the WLAN is unstable, and the rate is low. Therefore, it should not be used). ADSL can provide downward 8 MBPS bandwidth, that can satisfy the network computer applications that its requirement to the network bandwidth is not high [8]. Each market unified export and linked to the backbone network of the tender-invitation zone paying special attention to the ability of network switching equipment and the connection bandwidth of the Intranet network and backbone network when the client computers use the network computer, because the network computer needs the support of the larger network exchange capacity and network bandwidth. Because the distance of the whole market to the central office is less than $2 \mathrm{~km}$, single-mode fiber can reach the central office from the various markets without the relay amplify. Using single mode fiber, the bandwidth can be guaranteed, and the cost is low. 


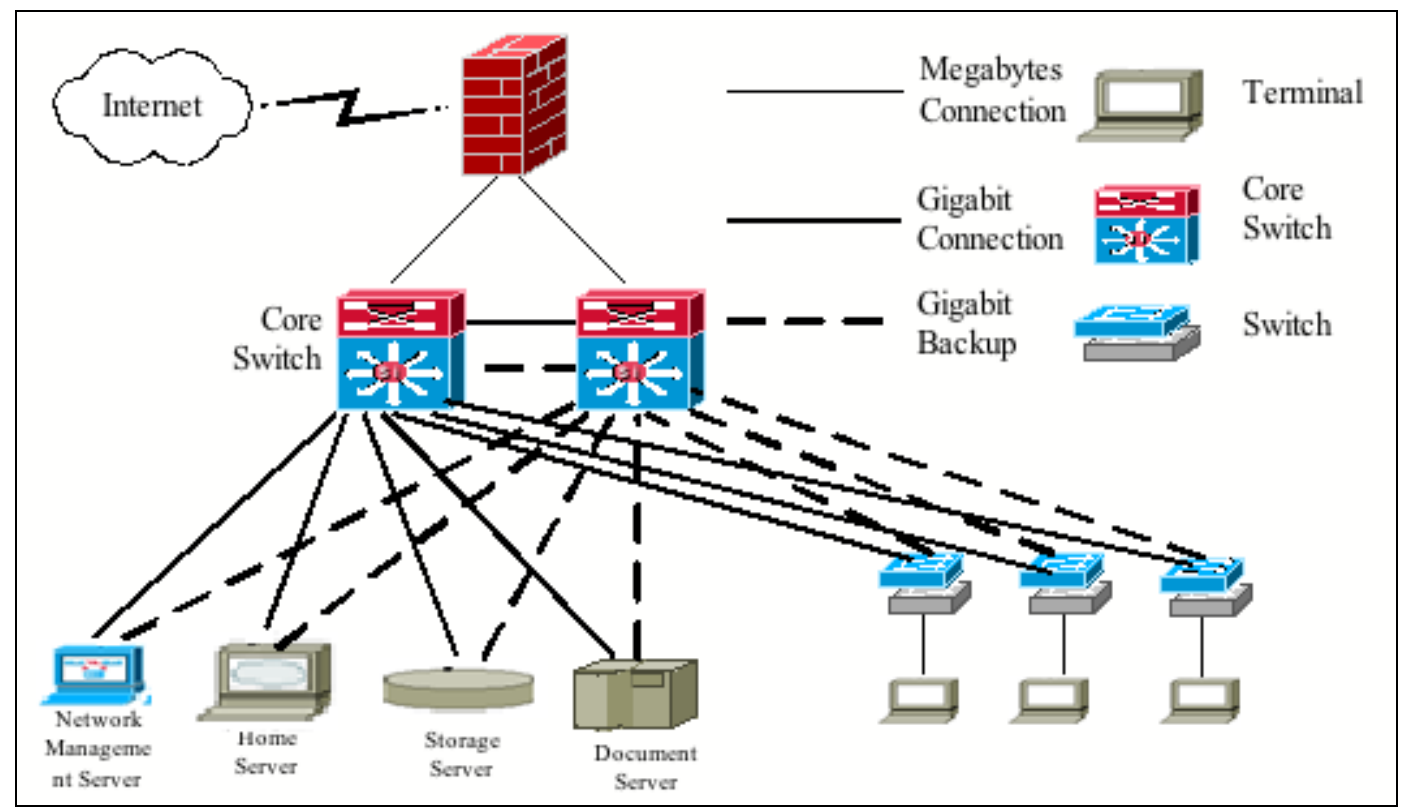

Fig. (4). Backbone network topology figure.

\subsection{Network Security Solutions}

E-commerce information system construction adopts multi-layer security, sets up security measures at the application layer, physical layer, logic layer, network layer and the other levels; control of the general IP address, subnet or domain name; the user name/password restrictions; use public key encryption to support the management to the multi-user operating powers [9].

\section{NETWORK SECURITY CONSTRUCTION OF THE APPAREL INDUSTRY E-COMMERCE PLATFORM}

The construction of apparel industry e-commerce platform system mainly adopts the following information security policy:

\subsection{Security and Service Done Together by the Hard- ware Equipment and Software}

Security software does not guarantee the absolute security of the operation and storage. When software runs, a lot of important information (such as keys and pins) will appear in a certain time clear in the computer's memory. Therefore, "high level" criminals stealing and taking advantage of these important information are very easy, so the system hardware and software should be used together to ensure safety.

\subsection{Practical and Reliable}

To fully meet the practical requirement of the system, using the mature technology and equipment, to ensure stability and reliability of the running environment, hardware and software.

\subsection{Integrated Operation System Design}

To ensure the security of the system, it not just depends on independent security equipment. We must consider from the perspective of system and do integration design to meet the demand of systematic safety. Specific measures are as follows:
(1) Set up the hardware protective wall: using router which has the function of firewall, realize preliminary safety for hardware level. The hardware protection wall after the router can separate the Intranet, the network and the demilitarized zone (DMZ), placed the servers that provide different services in different sections, opening the agreement which has the minimum requirements, and this is enough to prevent most of the hacker attacks.

(2) Using encryption protocol: system uses the SSL protocol (3.0) that is an international standard and it has been certified by the CA center. That realizes the client identity authentication and encryption transmission of the transaction information on the Internet, ensures that the trade request and the information on the site transmitted in a secure transmission channel. (SSL is a kind of data transmission protocol developed by NETSCAPE. That data transmits in the secure channel constructed between browser software which has the certificate and the WWW server. It runs between TCP/IP layer and application layer. Using TCP/IT on behalf of the senior agreement allows a WWW server that supports SSL to let itself get trust and let the client get the trust of the server on the client side in the process of data communication, consequently a reliable encrypted connection between two machines is established).

(3) Secure operating system: the server system uses a business operating system that is strong security; the operating system has many features. We can reasonably set the operating system combined with the actual need, and can ensure that the e-commerce system is a safe and reliable WEB site and it is difficult to break the WEB site [10].

(4) Software monitoring: adopting firewall with related monitoring software, install it on separate machines, that can set up a 24-hour dynamic safety monitoring and control system, do real-time monitoring on the important part of the web site (such as online bidding and disburses funds, etc.), cut off the hacker attack and illegal access and alarm in time, effectively ensure the security of e-commerce system daily operation. 
(5) Specification management: strict user management is the biggest key to eliminate hidden trouble. The user group and role were given different access scope and power. Remove all unnecessary username with security problems. Strict user password management, often changes important password. Stand-alone install antivirus software, does not open the emails from stranger and stranger address, regularly checks the computer virus, stay on high alert for network trap.

(6) Data backup: "data is assets; backup is the most important thing". In the enterprise what the most precious is not a variety of hardware, but the business data which much money cannot buy. If we cannot ensure the network data security, the large number of investment will lost its meaning. Redundant hardware is used to ensure the system backup continuously in the hardware level, such as faulttolerant methods. When a hardware failure or damage, backup hardware can work immediately to replace. That ensure the business to continue and the safety of data storage. Use tape and disk to save data and program code permanently, backup data and software, save the system data to other media, so when the system makes mistakes it can be restored to the backup state. It can completely prevent logic errors, because the backup medium and computer systems are separate, error will not copy to the medium. This means that as long as it keeps long enough historical data, it will be able to restore the correct data.

(7) Program control: in the program, pay attention to the management of the authority, adopt various methods to ensure that legitimate users can only access to the correct position and module. In the system, it uses the products based on the technology of JAVA component module. It can control the module, the table according to different groups, roles and people, and can control operations including access, read, add, modify, delete and other operations. Establish system logs, that can make the operation that covers the data changing be documented.

\subsection{Disaster Backup of Hardware System}

With the development of information construction, the development of the market is more and more dependent on computer systems, computer systems have to continuously provide data of production, marketing and management. Even a brief business interruption can also cause incalculable economic losses and reputation losses.

There are many factors that can affect the normal operation of the computer system, for some hardware factors, we can use some new technology to ensure the normal operation of the system, such as cluster and disaster tolerant. But for a number of factors, we can only through the way of data backup to prevent data loss, because when the system is interrupted what's the most important is to recover data in the shortest time, so we need to adopt an offline backup system to protect the data in the database [11].

\subsection{Automatic Network Storage}

The storage integration technology can help this storage system to realize the following functions:

1. Using multiple hosts connected storage technology to realize data integration.
2. SAN structure realizes high performance and the flexibility of architecture.

3. Resource management that crosses the architecture.

4. Snap view support the serves free backups, data extraction of the decision support system.

Using the automatic network storage to integrate information into the central data center means that all servers can access all information, regardless of where it is. Disaster recovery will be truly effective, business continuity also becomes possible. Soon it realizes information integration, and it also can use the copy software based on storage to realize "productivity protection" to manage and use automated software of all equipment, the cost of the related tasks will also drop.

\section{CONCLUSION}

Airport economic zone, directly or indirectly affects the economy of the surrounding areas, causing agglomeration effect and diffusion effect. It constructs electronic port information platform by using modern information technology help to realize electronic trading, improves transaction efficiency, reduces the transaction cost, promote the interaction of information flow, cash flow and logistics in the airport economic zone, and helps to promote the competitiveness of the enterprise. The article analyses the necessity of apparel industry e-commerce platform construction in airport economic zone, and then discusses the general structure and network topology structure of the e-commerce platform, analyses the design and construction of information platform, and finally discusses the main application system of the information platform. The platform application helps to strengthen the institutionalization and standardization of enterprise management, standardize the market economic order, and improve product quality and service quality, improve the competitiveness of the industry, enterprises and products in the economic zone.

\section{ABOUT THE AUTHOR}

Yang Changhui, Graduated from the Central South University, and now working at business school of Zhengzhou University, the main research directions include operation management, supply chain management and management information system.

\section{CONFLICT OF INTEREST}

The author confirms that this article content has no conflict of interest.

\section{ACKNOWLEDGEMENTS}

This paper was supported by NSFC (71272207, 71301150, U1304705, U1404704), NSSF 13BGL061, 10YJC630326 and 14YJC630126 (Humanity and Social Science Foundation of Ministry of Education).

This paper was supported by Science and Technology Research key project in Henan province department of education (No. 14A630053) and the humanity and social science research projects in Henan province department of education (2014-GH-057). 


\section{REFERENCES}

[1] G. Jongbloed, and G. Koole. "Managing uncertainty in call centers using Poisson mixtures", Applied Stochastic Models in Business and Industry, vol. 17, pp. 307-318, 2001.

[2] L. P. Ji, K. L. Luo, and H. Tan, "Based on three structures disperser -like call center system", Computer application, vol. 12, pp. 66-73, 2004.

[3] N. Prindezis, and C. T. Kiranoudis, "An internet-based logistics management system for enterprise chains", Journal of Food Engineering, vol. 70. no. 3, pp. 373-381, 2005.

[4] B. Yuan, T. Wang, and J. T. Wu, "An approach based componet for implementing a service-oriented information integration platform", International Journal of Organizational Innovation, vol. 2, no. 2, pp. 85-100, 2009.

[5] J. Santa, M.A. Zamora-Izquierdo, A.J. Jara, and A.F. Gomezskarmeta, "Telematic platform for integral management of agricultural/perishable goods in terrestrial logistics", Computers and Electronics in Agriculture, vol. 80, pp. 31-40, 2012.

[6] J. D. Nelson, and C. Mulley, "The impact of application of new technology on public transport service provision and the passenger experience: A focus on implement in Australia", Research In Transportation Economics, vol. 39, no. 1, pp. 300-308, 2013.

[7] J. S. Fu, Z. J. Zhang, X. L. Jin, and Z. Q. Hao, "Smart subway information platform based on internet of things", International Journal of Hybrid Information Technology, vol. 6, no. 1, pp. 177186, 2013.

[8] T. Levendovszky, A. Dubey, W.R. Otte, D. Balasubramanian, A. Coglio, S. Nyako, W. Emfinger, P. Kumar, A. Gokhale, and G. Karsai, "Distributed real-time managed systems: a model-driven distributed secure information architecture platform for managed embedded systems", IEEE Software, vol. 31, no. 2, pp. 62-69, 2014

[9] G. Divanis, A. Aonghusa, and P. Mac, "Privacy protection in open information management platforms", IBM Journal of Research \& Development, vol. 58, no. 1, pp. 1-11, 2014.

[10] S. Felix. and I. Provide, "Obtain and exchange information: the epublishing technology information platform CARPET", The UKSG journal, vol. 25, no. 3, pp. 305-310, 2012.

[11] K. Christian, D. J. Chen, and P. Jean-Michel, "Integrated analysis platform: an open-source information system for high-throughput plant phenotyping", Plant Physiology, vol. 165, no. 2, pp. 506-518, 2014.

(C) Yang Changhui; Licensee Bentham Open.

This is an open access article licensed under the terms of the Creative Commons Attribution Non-Commercial License (http://creativecommons.org/licenses/by-nc/3.0/) which permits unrestricted, non-commercial use, distribution and reproduction in any medium, provided the work is properly cited. 